Bert H. Herrmann, Georg Daeschlein, Sebastian von Podewils, Claudia Sicher, Jana

Kuhn, Kai Masur, Mareike Meister, Philip Wahl, Christoph Hornberger*

\title{
Detecting Bacteria on Wounds with Hyperspectral Imaging in Fluorescence Mode
}

\begin{abstract}
Chronic non-healing wounds represent an increasing problem. In order to enable physicians and nurses to make evidence based decisions on wound treatment, the professional societies call for supporting tools to be offered to physicians. Oxygen supply, bacteria colonization and other parameters influence the healing process. So far, these parameters cannot be monitored in an objective and routinely manner. Existing methods like the microbiological analysis of wound swabs, mean a great deal of effort and partly a long delay. In this paper 42 fluorescence images from 42 patients with diabetic foot ulcer, recorded with a hyperspectral imaging system $\left(\right.$ TIVITA $^{\circledR}$ ), converted for fluorescence imaging, were analysed. Beside the fluorescence images, information about the bacterial colonization is available from microbiological analysis of wound swabs. After preprocessing, principal component analysis, PCA, is used for data analysis with a 405 $\mathrm{nm}$ excitation wavelength, the emission wavelength range 510 $-745 \mathrm{~nm}$ is used for analysis. After dividing the data into a training and a test dataset it could be shown, that bacteria are detectable in the wound area. A quantification in bacterial colonization counts (BCC) was not in the focus of the research in this study stage.
\end{abstract}

Bert. H. Herrmann Hochschule Wismar, University of Applied Sciences, Technology, Business and Design, Faculty of Engineering, Wismar, Germany

Georg Daeschlein, Sebastian von Podewils, Claudia Sicher Department of Dermatology of the University Medicine Greifswald, Greifswald, Germany

Jana Kuhn, Clinic for Diabetes and Metabolic Diseases,

Karlsburg Hospital Dr. Guth GmbH \& Co KG, Karlsburg, Germany Leibniz Institute for Plasma Science and Technology, Greifswald, Germany.

Kai Masur, Mareike Meister, Leibniz Institute for Plasma Science and Technology, Greifswald, Germany.

Philip Wahl, Diaspective Vision GmbH, Pepelow, Germany.

${ }^{*}$ Corresponding author: Christoph. Hornberger: Hochschule Wismar, University of Applied Sciences, Technology, Business and Design, Faculty of Engineering, Philipp-Müller-Str. 14, 23966 Wismar, Germany, e-mail: Christoph.Hornberger@hs-wismar.de
Keywords: medical smear tests hyperspectral imaging, bacteria, infected wound, fluorescence, diabetic foot ulcer, principal component analysis PCA

https://doi.org/10.1515/cdbme-2020-3067

\section{Introduction}

The diabetic foot ulcer is one of the most common and most serious complications of diabetes mellitus and a common cause of the need for hospitalization in diabetics [1]. Between $2-10 \%$ of all diabetics suffer from a foot ulcer; the annual rate of new cases is $2.2-5.9 \%$ [2]. The chronic wounds underlying the diabetic foot ulcer, which are often accompanied by infections, lead to 43,000 amputations a year in Germany alone [Heller et al., 2005]. The limitations of the quality of life for the affected patients and the resulting health economic follow-up costs are immense.

Hyperspectral imaging (HSI) is widely used in medical applications within the last years [3-4]. In wound diagnostics, it has been successfully introduced for monitoring oxygenation and haemoglobin content in wound tissue [5-6]. Another parameter, which can negatively influence the healing process, is the bacteria load on wounds. With normal, remission HSI bacteria cannot be detected. But it is known, that bacteria show fluorescence emission when excited with UV light [7-8]. Examples of fluorescent substances in bacteria are Pyoverdin at Pseudomonas aeruginosa [9] and Pseudonomas syringae [10], Porphyrine at Staphylococcus aureus ssp. anaerobius [11] and Propionibacterium acnes \& Propionibacterium granulosum [12].

We propose a combination of HSI with UV excitation at $405 \mathrm{~nm}$ and analysing fluorescence light in the wavelength region $510 \mathrm{~nm}-745 \mathrm{~nm}$. Up to now 42 data cubes from patients with diabetic foot ulcer have been recorded with such a setup and microbiological swabs were performed in parallel. In some recordings fluorescence emission is directly visible in a RGB mode but in most images it is quite difficult to detect 
bacterial fluorescence with naked eyes. To improve the detectability of bacteria on wounds more sophisticated data analysis mechanisms are needed. After data pre-processing, we used principal component analysis (PCA) on a training dataset to identify bacteria. Three principal components proved to be characteristic for bacteria fluorescence. In this paper the technical setup, the data collection and data analysis is described and the preliminary results on 42 data cubes are presented.

\section{Materials and Methods}

\subsection{Technical HSI Setup}

The hyperspectral image cubes were obtained using a modified TIVITA $^{\circledR}$ camera (Diaspective Vision $\mathrm{GmbH}$, Pepelow, Germany) that was converted for fluorescence imaging using a $405 \mathrm{~nm}$ peak wavelength LED illumination. The measurement produces a three-dimensional data cube with the spatial dimensions of $640 \times 480$ pixels and one spectral dimension, the recorded fluorescence emission wavelength $\lambda$. The wavelength resolution is approx. $5 \mathrm{~nm}$ in the range from 500 to $1000 \mathrm{~nm}$, generating 100 spectral values. The emission was analysed in the $510-745 \mathrm{~nm}$ range.

\subsection{Clinical Data Acquisition}

Wound data were collected in a bi-centric, non-randomized, controlled cohort study at the University of Greifswald, Clinic and Polyclinic for Skin Diseases and the Competence Center Diabetes Karlsburg (Karlsburg Clinic for Diabetes and Metabolic Diseases and the Leibniz Institute for Plasma Science and Technology e.V. (INP), Greifswald).

Patients are recruited who are treated at the clinic Karlsburg for diabetic foot syndrome (DFS). At the Clinic for Skin Diseases at the University Medical Center Greifswald, patients are recruited who are treated for chronic ulcers of different genesis.

The data included up to now are 42 patients, 8 female and 34 male with an average age of 68,8 years ( \pm 8 years). 33 patients had a diabetic ulcer, the remaining 9 had a curis ulcer. 31 patients had peripheral artery disease, PAD. After successful inclusion, a photograph is taken of the ulcer, while the ulcer area is measured and loose crusts and deposits are removed by standard debridement with gauze without the application of tension or pressure. Heavily secreting ulcers must be patted dry briefly. Subsequently, a hyperspectral image examination is performed in which the measuring distance was kept constant at $50 \mathrm{~cm} \pm 2 \mathrm{~cm}$. Then a swab is rolled over the entire ulcer surface under slight pressure and brought to the microbiological laboratory of the Clinic and Polyclinic for Skin Diseases of the University Medical Center Greifswald for standard microbiological diagnostics and stored at room temperature until processing.

The microbiological processing is carried out according to national recommendations and results in findings with semiquantitative species identification of the different pathogens.

\subsection{Data Pre-Processing and PCA}

Up to now 42 hyperspectral data cubes have been recorded. The data were subdivided in a training dataset of 10 cubes and a test data set of 32 . For both datasets, the following preprocessing was conducted.

a $2^{\text {nd }}$ derivative computation followed by a standard normal variate transformation (SNV) and a data smoothing was executed on the wavelength axis.

- $\quad$ in the spatial dimension a moving average of $2 \times 2$ pixels was computed for noise reduction by keeping the image size of $640 \times 480$ pixels constant.

\subsection{Principal Component Analysis}

With the pre-processed training data the following data analysis steps were conducted:

- a rectangular section of the wound area was selected manually as region of interest.

- the spectral emission of the pre-processed data were saved in one file. This file contains all the data of each selected pixel of every training data cube. The spatial pixel mapping is destroyed in this way.

- we run a principal component analysis (PCA) on this spectral data file. With a weighting vector the $510-$ $745 \mathrm{~nm}$ wavelength band was selected for analysis. Overall 15 loadings were computed. Loadings are weights of the original variables to calculate the principal component. The loadings calculated with the training data were stored in a text file. For the computation, a virtual PC with 64 GB of RAM was used.

\subsection{Selection of Loadings and Computation of Pseudo Colour Images}

As described above, in some recordings, fluorescence emission is directly visible in a RGB mode but in most images, 
it is quite difficult to detect bacterial fluorescence. The problem is to select those loadings from the 15 computed loadings, which are most characteristic for bacteria fluorescence. Our goal was to find the three most bacteria characteristic loadings and to use them to compute a pseudo colour image. To select these three loadings we opened the pre-processed training data cubes and applied the loadings to the cubes, which means that the spectral vectors of each pixel are multiplied with the loading vectors. For each loading, a binary image was computed. For some of the images with clear fluorescence, bacteria fluorescence was visible. In this way, we selected the three most bacteria characteristic loadings manually in a successive manner. In a further step this manual part should be automated. With the three selected loadings, we compute a pseudo colour image, which shall help to identify bacteria on wounds. As the recorded hyperspectral image cubes differ in the dynamic, a further step is needed to find optimal limiting values for the pseudo colour image presentation in order not to over- or underexpose the images. The user should not have to deal with finding the best settings; the software should present him the bacteria image.

\subsection{Verification with Test Data}

We pre-processed the test data and computed pseudo colour images with the fixed loadings of the training data and with the fixed limiting values. An example is presented in figure 1. In the top part the RGB Image of a foot ulcer with visible fluorescence light (red) after excitation with a $405 \mathrm{~nm}$ $405 \mathrm{~nm}$ peak wavelength LED can be seen, in the bottom part the region, where bacteria are detected, is highlighted, the background is blackened. In some of the data cubes no region is highlighted which means that the algorithm did not detect bacteria. For all the 32 test data cubes we noted if there are bacteria detected and compared it with the results of the standard microbiological swab diagnosis. For some wounds, more than one microbiological swab was taken by a differential swabbing method and therefore regions without bacteria and regions with bacteria could be analysed within one image. The position, where the single swabs were taken, was marked in the study documentation, according to the fluorescence spots seen with the camera. In this way there are 47 samples for analysis.

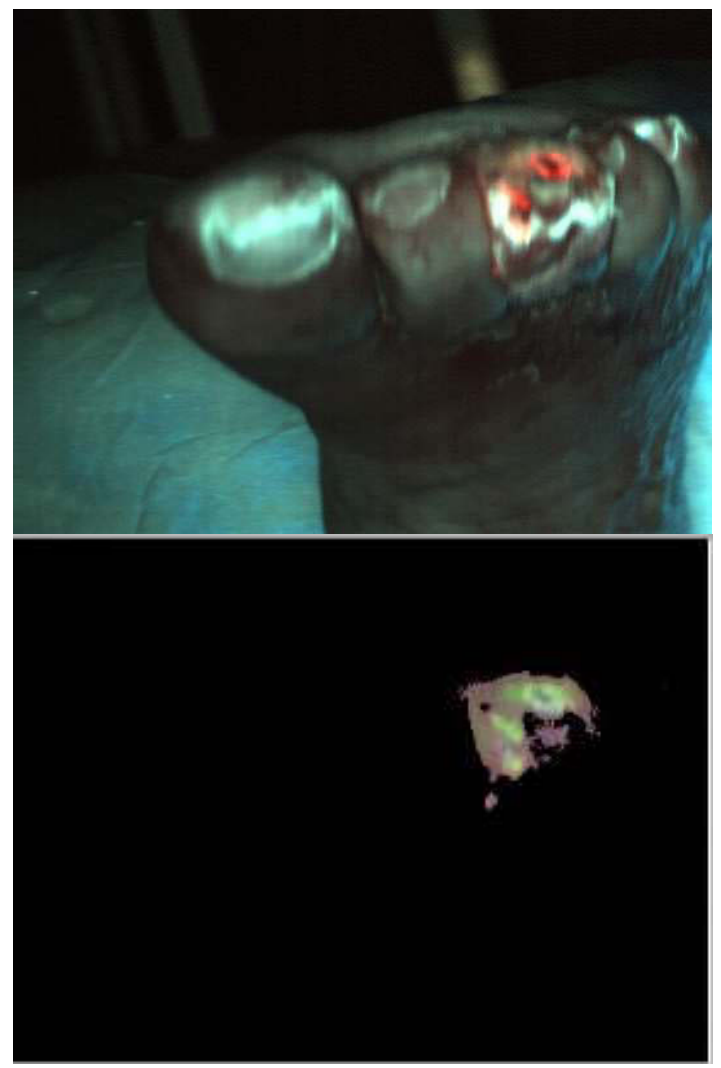

Figure 1: Top: The RGB Image of a foot ulcer with visible fluorescence light (red) after excitation with $405 \mathrm{~nm}$. Bottom: The region, where bacteria are detected is highlighted, the background is blackened

\section{Results}

The result of the comparison of the microbiological diagnosis of the smear tests and the bacteria detection of the algorithm is given in table 1 .

Table 1: Comparison of the microbiological test with the algorithm prediction of 32 cubes

\begin{tabular}{llll}
\hline & $\begin{array}{l}\text { Bacteria } \\
\text { detected by } \\
\text { Microbio- } \\
\text { logical Swab }\end{array}$ & $\begin{array}{l}\text { No Bacteria } \\
\text { detected by } \\
\text { Microbio- } \\
\text { logical Swab }\end{array}$ & Total \\
\hline $\begin{array}{l}\text { Bacteria } \\
\text { detected by }\end{array}$ & 24 & 0 & 24 \\
$\begin{array}{l}\text { Algorithm } \\
\begin{array}{l}\text { No Bacteria } \\
\text { detected by }\end{array}\end{array}$ & 5 & 3 & 8 \\
$\begin{array}{l}\text { Algorithm } \\
\text { Total }\end{array}$ & 29 & 3 & 32 \\
\hline
\end{tabular}


A sensitivity of $83 \%$ and a specificity of $100 \%$ is calculated. The result of the comparison of 47 different positions is given in table 2 . Here, a sensitivity of $81 \%$ and a specificity of $100 \%$ is calculated.

As there are only three cubes with a negative result of the microbiological diagnosis in the first analysis and five cubes in the second analysis, the specificity is not significant.

Table 2: Comparison of the microbiological test with the algorithm prediction of 47 samples

\begin{tabular}{llll}
\hline & $\begin{array}{l}\text { Bacteria } \\
\text { detected by } \\
\text { Microbio- } \\
\text { logical Swab }\end{array}$ & $\begin{array}{l}\text { No Bacteria } \\
\text { detected by } \\
\text { Microbio- } \\
\text { logical Swab }\end{array}$ & Total \\
\hline $\begin{array}{l}\text { Bacteria } \\
\text { detected by }\end{array}$ & 34 & 0 & 34 \\
$\begin{array}{l}\text { Algorithm } \\
\text { No Bacteria } \\
\text { detected by }\end{array}$ & 8 & 5 & 13 \\
Algorithm & & 5 & \\
Total & 42 & 5 & 47 \\
\hline
\end{tabular}

\section{Discussion}

Hyperspectral imaging in fluorescence mode with an excitation wavelength of $405 \mathrm{~nm}$ seems to be a promising tool for Bacteria detection. With 42 data cubes divided in 10 cubes for training an PCA algorithm and 32 test cubes a sensitivity of bacteria detection $>80 \%$ is yielded. Using all 57 microbiological swab analysis data, resulting in 47 test data a similar sensitivity was reached. A more detailed analysis of the PCA loadings suggest that in future a differentiation between different bacteria could be possible -e.g. a first differentiation between gram positive and gram negative bacteria. So far, there are not enough data for a well-founded statement. The data acquisition in the controlled study is still ongoing and we hope to improve the sensitivity of the method and will be able to show the recognition of bacterial types in the near future. The data acquisition will be adapted that way, that the position of the bacteria recognized by the algorithm should be used for targeted bacterial swabs at this position and beside this position to improve the verification process of the method.

\section{Author Statement}

Research funding: This work is funded by the European Regional Development Fund (ERDF or EFRE).
Conflict of interest: Authors state no conflict of interest. Informed consent: Informed consent has been obtained from all individuals included in this study.

Ethical approval: The research related to human use complies with all the relevant national regulations, institutional policies and was performed in accordance with the tenets of the Helsinki Declaration, and has been approved by the medical ethics committee (BB 040/19).

\section{References}

[1] Lobmann R: Diabetic Foot: how to avoid therapeutic mistakes. MMW Fortschr Med. 2013; 155(18):63-6

[2] Morbach S, Müller E, Reike H, Risse H, Rümenapf G, Spraul M: Diabetic foot syndrome. Exp Clin Endocrinol Diabetes 2014; 122(7):416-24

[3] Lu G. Fei B. Medical hyperspectral imaging: A review. J. Biomed. Optics 2014, 19(1) 010901 1-23. https://doi.org/10.1117/1.JBO.19.1.010901

[4] Chin MS, Freniere BB, Lo YC, Saleeby JH, Baker SP, Strom $\mathrm{HM}$, et al. Hyperspectral imaging for early detection of oxygenation and perfusion changes in irradiated skin. J. Biomed. Optics 2012, 17(2), 026010 1-5 https://doi.org/10.1117/1.JBO.17.2.026010

[5] Daeschlein G, Langner I, Wild T, von Podewils S, Sicher C, Kiefer T, Jünger M. Hyperspectral Imaging as a Novel Diagnostic Tool in Microcirculation of Wounds. Clinical Hemorheology and Microcirculation. 2017 67(3-4) 467-474. DOI: $10.3233 / \mathrm{CH}-179228$

[6] Yudovsky D, Nouvong A, Pilon L. Hyperspectral imaging in diabetic foot wound care. J. Diabetes Sci. 2010, Technol. 4(5), 1099-1113 https://doi.org/10.1177/193229681000400508

[7] Wu YC, Kulbatski I, Medeiros PJ, Maeda A, Bu J, Xu L, et al. Autofluorescence imaging device for real-time detection and tracking of pathogenic bacteria in a mouse skin wound model: preclinical feasibility studies. J. Biomed. Opt. 2014 19(8) 085002 1-9 https://doi.org/10.1117/1.JBO.19.8.085002

[8] Dartnell LR, Roberts TA, Moore G, Ward JM, Muller JP. Fluorescence Characterization of Clinically-Important Bacteria. PLoS ONE 2013 8(9):e75270. doi:10.1371/journal.pone.0075270

[9] Cox C, Adams P. Siderophore Activity of Pyoverdin for Pseudomonas aeruginosa. Infection and Immunity. 1985 48(1) $130-138$

[10] Cody YS, Gross DC. Characterization of PyoverdinpS5, the Fluorescent Siderophore Produced by Pseudomonas syringae pv. Syringae. Appl. And Environmental Microbiology, 1987, 53(5), 928-934

[11] De la Fuente R, Schleifer KH, Götz F and Köst HP. Accumulation of porphyrins and pyrrole pigments by Staphylococcus aureus ssp. anaerobius and its aerobic mutant. FEMS Mircrobiology Letters, 1986, 35, 183-188

[12] Lee WLS, Shalita AR, Poh-Fitzpatrick MB. Comparative Studies of Porphyrin Production in Propionibacterium acnes and Propionibacterium granulosum. Journal of Bacteriology 1978, 133(2), 811-815 